\title{
Impact of Provider Prior Use of HIE on System Complexity, Performance, Patient Care, Quality and System Concerns
}

\author{
Sue S. Feldman ${ }^{1}$ (D) Neset Hikmet ${ }^{2} \cdot$ Shikha Modi $^{1} \cdot$ Benjamin Schooley ${ }^{2}$ \\ Accepted: 9 September 2020 / Published online: 23 September 2020 \\ (C) Springer Science+Business Media, LLC, part of Springer Nature 2020
}

\begin{abstract}
To date, most HIE studies have investigated user perceptions of value prior to use. Few studies have assessed factors associated with the value of HIE through its actual use. This study investigates provider perceptions on HIE comparing those who had prior experience vs those who had no experience with it. In so doing, we identify six constructs: prior use, system complexity, system concerns, public/population health, care delivery, and provider performance. This study uses a mixed methods approach to data collection. From 15 interviews of medical community leaders, a survey was constructed and administered to 263 clinicians. Descriptive statistics and analysis of variance was used, along with Tukey HSD tests for multiple comparisons. Results indicated providers whom previously used HIE had more positive perceptions about its benefits in terms of system complexity $(p=.001)$, care delivery $(p=.000)$, population health $(p=.003)$, and provider performance $(p=.005)$; women providers were more positive in terms of system concerns $(p=.000)$; patient care $(p=.031)$, and population health $(p=.009)$; providers age 44-55 were more positive than older and younger groups in terms of patient care $(p=.032)$, population health $(p=.021)$, and provider performance $(p=.014)$; while differences also existed across professional license groups (physician, nurse, other license, admin (no license)) for all five constructs $(p<.05)$; and type of organization setting (hospital, ambulatory clinic, medical office, other) for three constructs including system concerns $(p=.017)$, population health $(p=.018)$, and provider performance $(\mathrm{p}=.018)$. There were no statistically significant differences found between groups based on a provider's role in an organization (patient care, administration, teaching/research, other). Different provider perspectives about the value derived from HIE use exist depending on prior experience with HIE, age, gender, license (physician, nurse, other license, admin (no license)), and type of organization setting (hospital, ambulatory clinic, medical office, other). This study draws from the theory of planned behavior to understand factors related to physicians' perceptions about HIE value, serving as a departure point for more detailed investigations of provider perceptions and behavior in regard to future HIE use and promoting interoperability.
\end{abstract}

Keywords Health information exchange $\cdot$ Medical informatics $\cdot$ Clinical informatics

\section{Introduction}

Health Information Exchange (HIE) has been described in various ways in the literature, but is generally understood as the act of health information sharing, facilitated by computing infrastructure, that is conducted across affiliated physicians' offices, hospitals, and clinics; or between completely disparate health systems (Furukawa et al. 2013). HIE across disparate

Sue S. Feldman

suefeldman1009@gmail.com

1 University of Alabama at Birmingham, 1716 9th Avenue So. SHPB 590K, Birmingham, AL 35294, USA

2 University of South Carolina, 550 Assembly Street, \#1300, Columbia, SC 29208, USA systems allows clinical information to follow patients as they move across different care settings, whether or not each organization shares an affiliation. This might include a hospital connected to an HIE that is, in turn, connected to other forprofit and not-for-profit hospitals, private practices, and clinics. HIE is expected to transform the nation's healthcare system through access to patient data from electronic health records to support care provision and coordination and improve care quality and population health (Berwick et al. 2008). Health information exchange has also been described in terms of the organizational and technical environment facilitating HIE. In this paper, HIE is the act of exchanging health information facilitated by a health information exchange organizational and technical environment.

While expectations and promises are high, still relatively little is known about the real and perceived value of HIE by 
providers, and how to accomplish large-scale acceptance and use. It has been reported that about two-thirds of hospitals and almost half of physician practices are now engaged in some type of HIE with outside organizations (Rahurkar et al. 2015). Relatively few of the more than 100 operational U.S. health information exchanges have been the subject of published evaluations (Rudin et al. 2014).

After more than a decade of HIE hype, utilization by users is still relatively low. A review of HIE past research indicated that most studies reported use of HIE in $2 \%$ to $10 \%$ of encounters (Rudin et al. 2014). Further, findings from a review of research on HIE sustainability suggest that just one quarter of existing HIE organizations consider themselves financially stable (Rudin et al. 2014). A systematic review of HIE studies suggests that study stakeholders claim to value HIE. Yet, the effects on a wide range of outcomes are still unknown (Rudin et al. 2014), and little generalizable evidence currently exists regarding sustainable benefits attributable to HIE (Rahurkar et al. 2015). Some have noted the potential for widespread HIE adoption to reduce the utilization and cost of healthcare services (Shapiro et al. 2011; Richardson 2001), though empirical evidence is limited (Bailey et al. 2013; Fontaine et al. 2010).

Continued research is needed to understand the factors associated with adoption and use of such a promising, yet underutilized technology. To date, most HIE studies have investigated user perceptions of value prior to use, and the intention to use. Few studies have assessed factors associated with the value of HIE through its actual use. This study investigates provider perspectives on HIE comparing those who had prior experience vs those who have only heard of HIE, but not yet had experience with it.

\section{Background}

The purpose of this study is to investigate provider perceptions about HIE, comparing those who have used HIE to those who have not used HIE and how perceptions differ. The objectives of this study are to explore demographic differences in perceptions across different types of providers, assessing several important factors related to the adoption of health IT.

Literature has determined that factors associated with perceived benefits and challenges of health IT adoption and use include: 1) the extent to which users perceive a system to be complex vs easy to use (Davis 1989; Gadd et al. 2011), 2) technical standards and business concerns that act as barriers to system use (Rudin et al. 2014), 3) the perceived effects that using a system (and its information) has on public or population health (Zech et al. 2015; Shapiro et al. 2011; Hincapie and Warholak 2011; Hessler et al. 2009; Dobbs et al. 2010), 4) the perceived effects that using a system (and its information) has on patient care delivery (Frisse et al. 2012; Furukawa et al.
2013; Kaelber and Bates 2007), and 5) the perceived effects that using a system (and its information) has on provider performance (Davis 1989). We review these factors below as well as the literature on prior use of an information system: or the effect of prior use vs nonuse on perceptions and expectations of a system.

\subsection{Prior Use}

Prior use of an information system has been found to be associated with the intention to use them in the future (Jackson et al. 1997; Agarwal and Prasad 1999). Prior IT usage behavior tends to be a significant determinant of future usage intention and/or behavior (Jasperson et al. 2005). Prior behavior helps form habit, which induces future behavior in an unthinking, automated, or routinized manner, rather than through a conscious process of cognitive or normative evaluation (Eagly and Chaiken 1993; Triandis 1977). Accordingly, future behavior can be viewed as a joint outcome of behavioral intention and habit. Though the indirect effects of prior use have seen little investigation in the literature, preliminary evidence to that effect has been reported (Taylor and Todd 1995a, b). These authors argued that as individuals gain familiarity with using a given technology (by virtue of prior use), they tend to focus less attention on the amount of effort needed to use the technology, and more on ways in which the technology can be leveraged to improve their job performance and outcomes. The familiarity with the technology gained from prior usage experience and the knowledge gained from learning-by-doing allows users to form a more accurate and informed opinion of the true performance benefits of the target system and its relevance to their job performance. Hence, users' performance expectancy tends to become stronger as their prior use of technology increases. We extend this line of reasoning for business information systems to the case of providers' use of health information technology, or in this case, HIE. HIE is particularly interesting as a unique and differing context from business due to the multi-organizational, distributed, and shared healthcare information technology setting.

\subsection{System Complexity}

Perceived system complexity, or the degree to which a person believes that using a particular information system would be difficult vs easy or free of effort, has long been used as a construct to assess user acceptance of information technologies (Davis 1989). Studies on ease of use have included a range of health information technologies including electronic health records (EHR) (Dünnebeil et al. 2012; Hennington and Janz 2007), telemedicine (Hu et al. 1999), clinical information systems (Paré et al. 2006), and others. Many studies on HIE ease of use have focused on the perceptions of prospective users not currently using HIE. In one such study, $74.4 \%$ of 
physicians interested in HIE, but not currently engaging in HIE, perceived that using HIE would be easy (Patel et al. 2011). In contrast, $37.1 \%$ of physicians not interested in HIE perceived that using HIE would be easy (Patel et al. 2011). In the past, ease of use of HIE has been positively predictive of system adoption and usage (Gadd et al. 2011), and has shown to impact successful retrieval of patient information that affected patient care (Genes et al. 2011). Of significant importance for this study is understanding perceived ease of use from users who have actually used HIE vs. those who have not.

\subsection{System Concerns}

Some past studies have found several concerns that providers have in terms of using HIE. A meta-analysis of 38 studies showed that stakeholders consider HIE to be valuable, but barriers include technical performance, workflow issues, and privacy concerns (Rudin et al. 2014). Concerns also include limits on the amount and type of information that providers want to use (Patel et al. 2011), and general fears of information overload from ill designed systems and/or utilization of duplicate or seemingly "competing" information systems (Rudin et al. 2014). For example, in one study, quality assurance (QA) reports generated by a health information exchange for medical practices was reported as the least valued system function due to skepticism about report validity and concerns that reports would reflect negatively on providers (Ross et al. 2010). Another study found that HIE usage was lower in the face of time constraints, questioning whether HIE may be considered an information burden rather than a help to users (Vest et al. 2011; Vest and Miller 2011). Time constraints, especially in primary and emergency care, have also tended to be a cause for concern in engaging in HIE. Mixed results from HIE evaluations have further raised concerns about its utility. For example, one study found that increased HIE adoption has been associated with reduced rates of laboratory testing for primary and specialist providers (Ross et al. 2013). Yet, in the same study, imputed charges for laboratory tests did not shift downward (Ross et al. 2013). This evidence led us to include health information exchange system concerns as an important construct for HIE provider perceptions and usage.

\subsection{Public/Population Health}

Prior studies have indicated the potential for HIE to aid in a range of population health and care coordination activities, care quality, and timely health maintenance and screening. In one study, HIE was shown to enable identification of specific patient populations, such as homeless (Zech et al. 2015), and those who have chronic or high risk health conditions (Shapiro et al. 2011). However, not all studies have showed significant results related to population and patient health outcomes (Hincapie and Warholak 2011). The authors in one study concluded that HIE usage was unlikely to produce significant direct cost savings, yet also noted that economic benefits of HIE may reside instead in other downstream outcomes such as better informed and higher overall quality care delivery (Ross et al. 2013). Broader HIE impacts include positive relationships with public health agencies (Hessler et al. 2009), improved public health surveillance (Dobbs et al. 2010), and increased efficiency and quality of public health reporting (Shapiro et al. 2011). Thus, we assess user perceptions of the impact of HIE on public/population health.

\subsection{Care Delivery}

The extent to which HIE impacts the delivery of patient care has been addressed in prior studies. More generally, the timely sharing of patient clinical information has been shown to improve the accuracy of diagnoses, reduce the number of duplicative tests, prevent hospital readmissions, and prevent medication errors (Frisse et al. 2011; Furukawa et al. 2013; Kaelber and Bates 2007; Yaraghi 2015; Eftekhari et al. 2017). For HIE specifically, usage has been associated with decreased odds of diagnostic neuroimaging and increased adherence with evidence-based guidelines (Bailey et al. 2013). These include timelier access to a broader range of patient interactions with the healthcare system (Unertl et al. 2013), improved coordination of care and patient health outcomes for human immunodeficiency virus patients (Shade et al. 2012), and positive patient perceptions of the impact on care coordination (Dimitropoulos et al. 2011). Providers continue to engage in HIE with the belief that care delivery will improve (Cochran et al. 2015).

Further, benefits have been noted for specific care settings. Emergency department access to HIE has been associated with a cost savings due to reductions in hospital admissions, head and body CT use, and laboratory test ordering (Frisse et al. 2011). Further, HIE has been associated with faster outside information access, and faster access was associated with changes in ED care including shorter ED visit length (52.9 min shorter), lower likelihood of imaging (by 2.5, 1.6, and 2.4 percentage points for CT, MRI, and radiographs, respectively), lower likelihood of admission (2.4\%), and lower average charges ( $\$ 1187$ lower) $(P \leq .001$ for all) (Everson et al. 2016). Provider perceptions about the positive effects HIE has on patient care delivery may be the strongest motivating factor for its adoption. For example, in one study, physicians most agreed that the potential benefits of HIE lie in care quality and were least worried about the potential for decreases in revenues resulting from the technology (Lee et al. 2012). A study that looked at perspectives of home healthcare providers indicated a decrease in ED referral rates with HIE (Vaidya et al. 2012). In another study, looking up clinical information (test 
results, clinic notes, and discharge summaries) on a patientby-patient basis was found to be the most valued function for HIE users, followed closely by the delivery of test results, for the care of patients (Ross et al. 2010). As such, perceived impact of HIE on care delivery is included as a dependent variable.

\subsection{Provider Performance}

One expected outcome of using HIE is that a performance improvement would occur. Perceived usefulness is defined here as "the degree to which a person believes that using a particular system would enhance his or her job performance." (Davis 1989) In sum, when a system is perceived to be useful, users believe positive performance will result. Prior HIE studies have shown that certain medical information is perceived to be more useful than others. One prior study found that physicians expressed agreement that HIE is useful for pathology and lab reports, medication information, and diagnoses with chief complaints (Lee et al. 2012). However, they expressed less agreement regarding the need for patient data items, functional test images and charts, care plans at the referring clinic/hospital, or duration of treatment (Lee et al. 2012). Different studies reported that providers expected HIE data would be useful to improve completeness and accuracy of patients' health records, efficiency with which clinical care is delivered, quality and safety of care, communication with other providers and coordination and continuity of care (Patel et al. 2011; Cochran et al. 2015). Prior studies have also indicated that quality patient information is believed to impact the elimination of duplicated medication as well as lab and imaging tests, prevention of drug-drug interactions, better decision making on the care plan and expedited diagnoses, and better ability to explain care plans to patients (Lee et al. 2012). Physicians have noted that data gaps, such as missing notes, adequacy of training (Cochran et al. 2015), and timely availability of information (Melvin et al. 2016) may pose a significant challenge to future HIE usage (Rudin et al. 2011). While these perceptions of usefulness are important relative to expected HIE use and resulting provider performance, we see expected and actual system use as scenarios that could potentially result in contrasting viewpoints. Perceptions of provider performance that result from actual use of HIE may provide a more real-to-life assessment.

\subsection{Research Question}

The objectives of this study are to explore demographic differences in perceptions across different types of providers, assessing several important factors related to the adoption of health IT. Our hypothesis is that provider age, gender, type of licensure (i.e., doctors, nurses), provider organizational setting (hospital, private practice), role in an organization (administration, patient care), and prior experience using HIE are factors that affect provider perceptions about HIE. For example, Lee and colleagues (Lee et al. 2012) found that different physician practice settings significantly influenced individual user perceptions. Based on this information, this study is addressing differences in provider perceptions for different HIE related constructs described above.

\section{Methods}

The above research question was empirically tested using a field survey of practicing health providers in the state of Virginia, USA. The specific technology examined was health information exchange and the action examined was HIE.

\subsection{Study Setting}

In March 2010, the Office of the National Coordinator for Health IT (ONC) awarded a state cooperative agreement to the Virginia Department of Health (VDH) to govern statewide HIE. In September 2011, Community Health Alliance (CHA) was awarded a contract from VDH to build the Virginia Statewide health information exchange; ConnectVirginia was subsequently initiated to accomplish this goal. Statewide health information exchanges were regarded as an organizational structure to provide a variety of mechanisms to enable HIE using standardized technologies, tools, and methods. During the 27-month study period, ConnectVirginia designed, tested, developed, and implemented three technical exchange services: ConnectVirginia DIRECT Messaging (a secure messaging system), ConnectVirginia EXCHANGE (the focus of this study), and a Public Health Reporting Pathway. ConnectVirginia EXCHANGE is a query/retrieve service in which a deliberate query passively returns one or more standardized continuity of care documents (CCDs) that provide a means of sharing standardized health data between organizations on-boarded and connected to ConnectVirginia.

The health information exchange design was based on a secure means to exchange patient information between providers via DIRECT messaging, a secure means for query and retrieval of patient information via EXCHANGE, and a secure means for public health reporting. Consistent with other health information exchange developments, a standardized product development lifecycle was used to create and implement the system. ConnectVirginia's DIRECT and EXCHANGE protocols were originally established by ONC and used as a method to standardize HIE of secure messages and CCDs. Similar to the original Nationwide Health Information Exchange (NwHIN) Connect's EXCHANGE, ConnectVirginia's EXCHANGE involves pulling information by providers from an unfamiliar healthcare facility. ConnectVirginia's DIRECT 
is a simplified version of the Connect software that allows simple exchange of basic information between providers (Dimick 2010). The public health reporting pathway was established using secure file transport protocols.

\subsection{User Survey}

A survey questionnaire was developed and administered to physicians, dentists, nurse practitioners, registered nurses, physician assistants, and nurse midwives in Virginia. We evaluated user perceptions of the HIE using selected survey items. Each clinician was given a summary of the study, the study protocol, consent procedure, and were notified that the research had been approved by the institutional review board of the lead researcher's university. Each participant consented to participate and was assured their responses would be anonymous. The survey had three sections: (1) demographics (age, job, gender) and system usage characteristics; (2) familiarity with technology; and (3) user perceptions across an author generated scale inclusive of the following constructs: system complexity, health information exchange system concerns, provider performance, patient care, and population care. A panel of experts reviewed items for face validity. We collected responses for all items on a scale of 1 to 4 from strongly agree (1) to strongly disagree (4). Participants could also leave comments in several sections.

\subsection{Sampling and Subject Recruitment}

Purposive sampling was used to invite individuals, key informants, and thought leaders in the medical community to participate in 60-min interviews. Fifteen interviews were conducted. From these interviews, a survey was created and administered to physicians, dentists, nurse practitioners, registered nurses, physician assistants, and nurse midwives. The sample was achieved from multiple sampling sources including two state Medical Societies and through a Virginia medical providers e-Rewards panel. e-Rewards, a Research Now company, is one of the leading providers of online sampling in the U.S. product research industry. Surveys were conducted via two channels: telephone and Internet. e-Rewards surveys were conducted by experienced telephone surveyors. In order to avoid survey bias, online and telephone surveys rotated questions. The goal was to achieve 250 usable responses. Over a 4-week period, invitations to participate in the survey and the survey link were distributed in monthly newsletters to qualified members of the Medical Society of Virginia (11,000 members) and the Old Dominion Medical Society. Old Dominion Medical Society did not disclose the total number of members. This resulted in 125 surveys, of which 113 were usable. E-Rewards panel members were called until 150 completed and usable surveys were accomplished. Combined, this yielded in 263 usable surveys.

\subsection{Data Collection and Analysis}

IBM SPSS Data Collection was used to create and administer the survey online. Data were collected from May through June 2012. All surveys collected through telephone were entered into the online survey system as responses were collected. Data were analyzed from the online and telephone versions of the survey together. Internal reliability of the scale was calculated using Cronbach's alpha and used the statistical package IBM SPSS v. 22 for quantitative analyses. Descriptive statistics were compared across demographics and usage characteristics. Data were summarized using mean, median, and SD, and a sign test was used to determine if individual subscale items were significantly different from neutral. To determine the effects of our independent variables (prior use, gender, age, professional license, role in the organization, type of organization) on our dependent outcomes, an analysis of variance (ANOVA) was used along with Tukey HSD tests for multiple comparisons.

\subsection{Construct Measurement}

The five constructs of interest to this study were health information exchange system complexity, system concerns, public/ population health, care delivery, and provider performance. Each construct was measured using multiple-item survey questions, adapted from prior research, and reworded to reflect the current context of providers HIE usage. The complete item scales are provided in Table 1 . System complexity was measured using four Likert-scaled items adapted from Davis' perceived usefulness scale (perceived usefulness is also referred to as performance expectancy in the information technology usage literature) (Davis 1989). Perceptions about system concerns were measured using items modified from Taylor and Todd (Taylor and Todd 1995a, b). The effect of HIE on patient care delivery, public/population health, and provider performance was measured using investigator developed Likert-scaled items guided by literature review. Prior HIE usage was measured using three items similar to Thompson et al. (Thompson et al. 1994) that asked subjects whether they had previously or currently used the system. We did not have access to actual system-recorded usage data, and thus, self-reported usage data was employed as a proxy for actual recorded usage. Since the usage items were in the "yes/ no" format, in contrast to Likert scales for other perceptual constructs, common method bias was not expected to be significant. We assess demographic factors including participant gender, age, and professional license (physician, nurse, other, administrative); role in the organization (patient care, administration, teaching/research, other); and type of organization (hospital, ambulatory clinic, medical office, other). These were all determined via selectable items in the instrument. 
Table 1 Demographics

Previous HIE Use (N, \%)

\begin{tabular}{|c|c|c|c|c|}
\hline & \\
\hline & Prior HIE use & No HIE use & Total & $\%$ \\
\hline \multicolumn{5}{|l|}{ Age Group } \\
\hline 34 and below & 21 & 1 & 22 & 8.37 \\
\hline 35 to 44 & 44 & 11 & 55 & 20.91 \\
\hline 45 to 54 & 56 & 18 & 74 & 28.14 \\
\hline 55 to 64 & 72 & 22 & 94 & 35.74 \\
\hline 65 and over & 14 & 4 & 18 & 6.84 \\
\hline Total & 207 (78.7) & $56(21.3)$ & 263 & 100.00 \\
\hline \multicolumn{5}{|l|}{ Role in the Organization } \\
\hline Patient Care & 128 & 38 & 166 & 63.12 \\
\hline Administration & 47 & 17 & 64 & 24.33 \\
\hline Teaching / Research & 21 & 0 & 21 & 7.98 \\
\hline Other & 11 & 1 & 12 & 4.56 \\
\hline Total & 207 & 56 & 263 & 100.00 \\
\hline \multicolumn{5}{|l|}{ Type of Organization } \\
\hline Hospital (including academic) & 74 & 10 & 84 & 31.94 \\
\hline Ambulatory Clinic & 27 & 13 & 40 & 15.21 \\
\hline Medical Office (Private Practice) & 88 & 22 & 110 & 41.83 \\
\hline Other & 18 & 11 & 29 & 11.03 \\
\hline Total & 207 & 56 & 263 & 100.00 \\
\hline \multicolumn{5}{|l|}{ Professional License } \\
\hline Doctor & 97 & 26 & 123 & 48.81 \\
\hline Nurse & 79 & 12 & 91 & 36.11 \\
\hline Other Licensed Professionals & 22 & 16 & 38 & 15.08 \\
\hline Total & 198 & 54 & 252 & 100.00 \\
\hline \multicolumn{5}{|l|}{ Gender } \\
\hline Male & 69 & 25 & 94 & 37.75 \\
\hline Female & 125 & 30 & 155 & 62.25 \\
\hline Total & 194 & 55 & 249 & 100.00 \\
\hline
\end{tabular}

\subsection{Validation}

Each of the five constructs were tested for their reliability, or the extent to which each represents a consistent measure of a concept. Cronbach's alpha was used to measure the strength of consistency. Results of validation testing were found to be strong for patient care (0.82), provider performance (.90), and population care (.78); and moderate for system complexity (.62) and system concerns (.64).

\section{Results}

Among responses, 207 physicians indicated they had used HIE previously and the 56 remaining physicians indicated they had not yet used HIE. Respondents represented all clinical specialties, including internal medicine, pediatrics, gynecology, pathology, general surgery, anesthesiology, radiology, neurology, oncology, and cardiology. Selected sample demographics, along with the population demographics for all 263 providers at this hospital (obtained directly from the hospital administration), are shown in Table 1.

A one-way between subject's ANOVA was conducted to compare statistical differences across demographic categories on each previously identified construct. Demographic categories included: whether the participant had previously engaged with HIE or not, gender, age grouping, professional license, role in the organization, and type of organization. Constructs previously identified included health information exchange complexity, health information exchange system concerns, benefits of HIE on patient care, population health, and provider performance. Results from ANOVA are shown in Table 2.

Results indicated that there was a statistically significant difference between those that had previously used HIE and those that had not used HIE for four constructs. These included provider beliefs about the complexity $(\mathrm{F}(1,261)=10.336$, 
Table 2 Single table ANOVA

ANOVA

\begin{tabular}{lllllll}
\hline Prior HIE Use (Y/N) & Prior HIE Use p (F) & Age $p(\mathrm{~F})$ & Gender $\mathrm{p}(\mathrm{F})$ & Prof License p(F) & Role in Org p(F) & Type of Org p(F) \\
\hline System Complexity & $.001(10.336)$ & $.266(1.332$ & $.074(3.220)$ & $.003(4.818)^{*}$ & $.668(.522)$ & $.075(2.323)$ \\
System Concerns & $.997(.000)^{*}$ & $.389(.948)$ & $.000(30.413)^{*}$ & $.000(15.927)^{*}$ & $.647(.553)$ & $.017(3.438)^{*}$ \\
Care Delivery & $.000(14.765)^{*}$ & $.032(3.474)^{*}$ & $.031(4.731)^{*}$ & $.000(6.186)^{*}$ & $.135(1.871)$ & $.245(1.395)$ \\
Pop Health & $.003(9.241)^{*}$ & $.021(3.944)^{*}$ & $.009(6.989)^{*}$ & $.018(3.406)^{*}$ & $.551(.702)$ & $.018(3.399)^{*}$ \\
Provider Perform. & $.005(7.992)^{*}$ & $.014(4.349)^{*}$ & $.110(2.577)$ & $.008(4.000)^{*}$ & $.914(.174)$ & $.018(3.434)^{*}$ \\
\hline
\end{tabular}

*The mean difference is significant at the 0.05 level

$p=.001)$, perceived benefits on patient care $(\mathrm{F}(1,261)=$ $14.765, p=.000)$, perceived benefits on population health $(\mathrm{F}(1,261)=9.241, p=.003)$, and perceived benefits on provider performance $(\mathrm{F}(1,261)=7.992, p=.005)$, with providers that had previously used HIE showing more positive responses in each category Table 2 .

Statistically significant differences between gender groups were found for three constructs including provider system concerns $(\mathrm{F}(1,247)=30.413, p=.000)$; and perceived benefits on patient care $(\mathrm{F}(1,247)=4.731, p=.031)$, and on population health $(\mathrm{F}(1,247)=6.989, p=.009)$. Statistically significant differences between age groups were found for three constructs including provider perceived benefits on patient care $(\mathrm{F}(2,260)=3.474, p=.032)$, population health $(\mathrm{F}(2$, $260)=3.944, p=.021)$, and provider performance $(\mathrm{F}(2$, $260)=4.349, p=.014)$. Statistically significant differences between professional license groups (physician, nurse, other license, admin (no license)) were found for all five constructs, including provider perceptions about system complexity $(\mathrm{F}(3$, $259)=4.818, p=.003)$, provider concerns about use $(\mathrm{F}(3$, $259)=15.927, p=.000)$, provider perceived benefits on patient care $(\mathrm{F}(3,259)=6.186, \mathrm{p}=.000)$, population health $(\mathrm{F}(3$, $259)=3.406, p=.018)$, and provider performance $(\mathrm{F}(3$, $259)=4.000, p=.008)$.

Statistically significant differences between type of organization setting (hospital, ambulatory clinic, medical office, other) were found for three constructs including provider concerns about use $(\mathrm{F}(3,259)=3.438, p=.017)$, and provider perceived benefits on population health $(\mathrm{F}(3,259)=3.399$, $\mathrm{p}=.018)$, and on provider performance $(\mathrm{F}(3,259)=3.434$, $\mathrm{p}=.018)$. There were no statistically significant differences found between groups based on a provider's role in an organization (patient care, administration, teaching/research, other).

\subsection{Post Hoc Analysis: Tukey Honest Significance Difference (HSD) Test}

An ANOVA test is important to assess the significance of results; however, an ANOVA test does not provide information about where the statistically significant differences lie for multiple comparisons (groupings for age, licensure, type of organization). In order to analyze which specific group means are different, Tukey's HSD test is conducted for ANOVA results with statistically significant F-values (Tukey 1949).

Tukey HSD post hoc comparisons indicated that the mean score for participants between 40 to 55 years old $(\mathrm{M}=1.62$, $\mathrm{SD}=0.57$ ) was significantly different from participants over 55 years old $(\mathrm{M}=1.82, \mathrm{SD}=0.52)$ for provider perceived benefits on patient care. These two age groups, respectively, were also significantly different in terms of perceived benefits on population health $(\mathrm{M}=1.81, \mathrm{SD}=0.75)$ vs $(\mathrm{M}=2.07$, $\mathrm{SD}=0.64)$, and provider performance $(\mathrm{M}=1.96, \mathrm{SD}=0.58)$ vs $(\mathrm{M}=2.20, \mathrm{SD}=0.55)$.

Results from Tukey HSD post hoc tests of professional license groups on system complexity resulted in participants who identified as nurses $(\mathrm{M}=1.83, \mathrm{SD}=0.57)$ being significantly different from those identifying as other licensed professionals $(M=2.29$, $\mathrm{SD}=0.52)$; while nurses $(\mathrm{M}=3.18$, $\mathrm{SD}=0.50)$ were also different from physicians $(\mathrm{M}=2.60$, $\mathrm{SD}=0.70$ ) in terms of system concerns about HIE use. Analysis of perceptions about HIE benefits resulted in nurses $(\mathrm{M}=1.82, \mathrm{SD}=0.58)$ being significantly different from other licensed professionals $(\mathrm{M}=2.24, \mathrm{SD}=0.67)$ in their beliefs about the positive impacts on population health, and nurses $(\mathrm{M}=1.95, \mathrm{SD}=0.51)$ being different from other licensed professionals $(\mathrm{M}=2.33, \mathrm{SD}=0.54)$ in their perspectives about the benefits on provider performance. Finally, for perspectives on HIE benefits on patient care, other licensed professionals $(\mathrm{M}=2.04, \mathrm{SD}=0.56)$ were found to be significantly different from nurses $(\mathrm{M}=1.60, \mathrm{SD}=0.47)$ and from physicians $(\mathrm{M}=$ $1.73, \mathrm{SD}=0.60)$, with nurses being most positive and other professionals being least positive between the groups.

Results from Tukey HSD post hoc tests on type of organization indicated that participants whose primary work affiliation is with hospitals $(\mathrm{M}=2.89, \mathrm{SD}=0.66)$ were significantly different than those who identified with medical offices/ private practice $(\mathrm{M}=2.70, \mathrm{SD}=0.67)$ in terms of their concerns with using HIE. Results showed these two groups were 
also significantly different in terms of HIE benefits on provider performance $((\mathrm{M}=1.96, \mathrm{SD}=0.57)$ vs. $(\mathrm{M}=2.20, \mathrm{SD}=$ 0.53 ), respectively). Hospital based providers were less concerned and more positive towards performance benefits. Results also indicated that ambulatory clinics $(M=1.72$, $\mathrm{SD}=0.62$ ) were significantly different from medical offices/ private practices $(\mathrm{M}=2.10, \mathrm{SD}=0.75)$ in their beliefs that HIE benefits population health.

\section{Discussion}

The primary goal of this study was to identify differences in providers' perceptions related to HIE based on their demographics and prior use of HIE. A field survey was created based on provider interviews and administered to providers from different disciplines. The findings indicate that there were statistically significant differences in most HIE perceptions based on demographics including prior HIE use, gender, age, professional license, and type of organization. Professional role in an organization yielded no statistically significant differences.

The providers that had previously used HIE showed more positive responses towards HIE in each category except for the system concerns category. These results seem to support the business literature indicating that prior use of an information system has been shown to be associated with the intention to use it in the future, regardless of the amount of effort needed to use the technology (Jackson et al. 1997; Agarwal and Prasad 1999). There was a statistically significant difference in perceptions related to system complexity, system concerns, patient care, and population care between different genders, with females generally showing more positive responses than males for each category. Depending on age groups, a statistically significant difference was observed for the patient care, population care, and provider performance constructs. Interestingly, the middle group (ages 40 to 55) showed more positive responses than both the younger providers (less than 40 years) and older providers (over 55 years) for each category. While other studies have not looked at age by these constructs, this finding differs from literature suggesting that those 45 years and younger are more likely to adopt EHRs (Decker et al. 2012). It could be that the HIE context may be perceived differently than EHRs due to it being connective to and enabling of interoperability between EHRs. It could also be the size of the sample for each age group. In this sample, we have more providers that are in that middle age group compared to those that belong to the younger and older age groups. Another potential way to explain this finding could be using the diffusion of innovation theory (Kaminski 2011). Younger providers and older providers could be part of the later majority (conservatives) and may be less than enthusiastic at the beginning, as they could be waiting on statistically significant evidence before adopting and implementing new technology. The middle age group could be part of the visionaries or pragmatists as described in the diffusion of innovation theory and may be willing to be the trail blazers or risk takers (Kaminski 2011). Statistically significant differences for all five constructs were noted across all professional license groups, which indicates that providers have different perceptions about HIE depending on their discipline. In general, nurses reported the lowest scores about perceived complexity of HIE, yet the highest scores in terms of system concerns. Other licensed professionals reported the highest scores (high perceptions about HIE task complexity). Nurses and administrators reported the most positive scores on the benefits of HIE for patient care, population health, and provider performance. Doctors reported the least amount of concern with using HIE, while nurses reported the highest amount of concern. Statistically significant differences were noted for system concerns, population health, and provider performance constructs between different types of organizational groupings. The hospital based and "other organization" type groupings reported the least concerns about HIE use and those working for medical offices reported the highest amount of concern. Participants who reported working within ambulatory clinics reported the highest perceived benefit of HIE on population health while those working within medical offices/private practice reported the lowest perceived benefit and this is consistent with the literature across a variety of ambulatory settings (Haidar et al. 2017). Participants who reported working within hospitals reported the highest perceived benefit of HIE on provider performance while those working in medical offices/private practice reported the lowest perceived benefit.

\section{Limitations of the Study}

The findings of this study should be interpreted in light of its limitations. The first limitation is our measurement of the prior HIE usage behavior construct. Our self-reported measure of usage was not as accurate, unbiased, or objective as usage data from system logs. We urge future researchers to use system log-based measures of IT usage, if available.

Second, our small sample size, and correspondingly low statistical power, may have contributed to our inability to observe significant effects of prior usage behavior on each construct. We encourage future researchers to consider using larger samples, such as by using pooled observations from two or more hospitals and/or other healthcare facilities. Third, there were 14 participants that did not select either male or female gender. Thus, the analysis of gender did not include the entire sample.

Finally, there may be additional factors beyond those examined in this study. Our choice of the factors used here was motivated by the HIE literature and a first round of interviews 
with providers. However, there may be other theories, such as innovation diffusion theory or political theory, that may also be relevant to explaining provider perceptions and behavior. Future studies can explore those theories for identifying other predictors of provider behavior and/or compare the explanatory ability of those theories discussed herein.

\section{Implications for Practice}

The findings of this study have interesting implications for health IT practitioners. First, we provide evidence of the perspectives of various types of providers in terms of their beliefs and perceptions about HIE. Perceptions about system complexity, system concerns, patient care, population health, provider performance, and prior usage have varying effects in terms of influencing provider' perceptions about HIE. However, implementing standards of care that incorporate $\mathrm{HIE}$ is an instance of organizational change, requiring careful planning and orchestrating of change management to influence providers to routinely use the targeted technologies. Change management programs designed to enhance provider intentions to use health IT should focus on educating users on the expected performance gains from technology usage as well as improving their perceptions of behavioral control by training users to use those technologies appropriately.

The significance of prior behavior on future HIE usage intention is indicative of the importance of recruiting early adopters to "seed" HIE usage in hospitals and healthcare settings. Junior practitioners, by virtue of their more recent medical training involving the latest health IT, may be viewed as more likely to be such early adopters. However, the results of this study also show that providers age 40 to 55 may provide a strong base of supporters. Given their prior usage behavior and correspondingly, higher level of comfort with such technologies, these individuals are likely to continue using HIE further in hospital settings, even when other conditions may be less conducive to their usage. With major health IT policy efforts focused on interoperability, this study contributes to the perspective that different provider groups may be stronger facilitators of interoperability efforts than others and thus these findings could help managers and policymakers determine strategies for such efforts.

\section{Implications for Research}

This study examined provider perceptions about HIE, comparing those who have not used HIE with those who have previously used the technology. As such, the role of prior behavior on providers' perceptions and intentions regarding future HIE usage provides new insights. One may hypothesize that if individuals use a system that is perceived to provide value, then use of that technology will proliferate and expand throughout the intended user population. Findings indicate that this may not always be the case. As systems become more integrated, inter-organizational, and more complex, user perceived value derived from using that system may not be understood by the user. This study contributes to the nascent stage of theorizing in the medical informatics literature by presenting the theory of planned behavior as a referent theory that can not only help us understand providers' usage of HIE better, but can also serve as a starting point for more detailed investigations of provider behavior.

Second, given that the centrality of HIE usage to improving healthcare delivery, quality, and outcomes and the uphill battle many states and regions are currently facing to get providers to use HIE, our study provides some preliminary suggestions about how providers' behaviors can be influenced using a strong evidence base. We also elaborate the contingent role played by prior HIE usage experience in shaping providers' usage patterns. Presumably, there may be more such contingent factors that may be the subject of future investigations. In conclusion, we hope that this study will motivate future researchers to examine in further depth provider HIE usage behavior and contribute to a cumulative body of research in this area.

\section{Compliance with Ethical Standards}

Conflict of Interest On behalf of all authors, the corresponding author states that there is no conflict of interest.

\section{References}

Agarwal, R., \& Prasad, J. (1999). Are individual differences germane to the acceptance of new information technologies? Decision Sciences, 30(2), 361-391.

Bailey, J. E., Wan, J. Y., Mabry, L. M., Landy, S. H., Pope, R. A., Waters, T. M., et al. (2013). Does health information exchange reduce unnecessary neuroimaging and improve quality of headache care in the emergency department? Journal of General Internal Medicine, 28(2), 176-183.

Berwick, D. M., Nolan, T. W., \& Whittington, J. (2008). The triple aim: Care, health, and cost. Health Affairs, 27(3), 759-769.

Cochran, G. L., Lander, L., Morien, M., Lomelin, D. E., Sayles, H., \& Klepser, D. G. (2015). Health care provider perceptions of a querybased health information exchange: Barriers and benefits. Journal of Innovation in Health Informatics, 22(2), 302-308. https://doi.org/ 10.14236/jhi.v22i2.135.

Davis, F. (1989). Perceived usefulness, perceived ease of use, and user acceptance of information technology. MIS Quarterly, 13(3), 319340 .

Decker, Sandra L., Eric W. Jamoom, and Jane E. Sisk. (2012). Physicians in nonprimary care and small practices and those age 55 and older lag in adopting electronic health record systems. Health Affairs 31(5), 1108-1114.

Dimick, C. (2010). NHIN direct: ONC keeps it simple in effort to jumpstart data exchange. Journal of AHIMA, 81(6), 30-34. 
Dimitropoulos, L., Patel, V., Scheffler, S. A., \& Posnack, S. (2011). Public attitudes toward health information exchange: Perceived benefits and concerns. American Journal of Managed Care, 17, SP111SP116.

Dobbs, D., Trebatoski, M., \& Revere, D. (2010). The northwest public health information Exchange's accomplishments in connecting a health information exchange with public health. Online Journal Of Public Health Informatics, 2(2). https://doi.org/10.5210/ojphi. v2i2.3210.

Dünnebeil, S., Sunyaev, A., Blohm, I., Leimeister, J. M., \& Krcmar, H. (2012). Determinants of physicians' technology acceptance for ehealth in ambulatory care. International Journal of Medical Informatics, 81(11), 746-760.

Eagly, A. H., \& Chaiken, S. (1993). The psychology of attitudes. New York: Harcourt Brace Jovanovich College Publishers.

Eftekhari, S., Yaraghi, N., Singh, R., Gopal, R. D., \& Ramesh, R. (2017). Do health information exchanges deter repetition of medical services? ACM Transactions on Management Information Systems (TMIS), 8(1), 1-27.

Everson, Jordan, Keith E. Kocher, and Julia Adler-Milstein. (2016). Health information exchange associated with improved emergency department care through faster accessing of patient information from outside organizations. Journal of the American Medical Informatics Association, 24.e1, e103-e110

Fontaine, P., Ross, S. E., Zink, T., \& Schilling, L. M. (2010). Systematic review of health information exchange in primary care practices. The Journal of the American Board of Family Medicine, 23(5), 655-670. https://doi.org/10.3122/jabfm.2010.05.090192.

Frisse, M. E., Johnson, K. B., Nian, H., Davison, C. L., Gadd, C. S., Unertl, K. M., et al. (2011). The financial impact of health information exchange on emergency department care. Journal of the American Medical Informatics Association, 19, 328-333. https:// doi.org/10.1136/amiajnl-2011-000394.

Frisse, M. E., Johnson, K. B., Nian, H., Davison, C. L., Gadd, C. S., Unertl, K. M., et al. (2012). The financial impact of health information exchange on emergency department care. Journal of The American Medical Informatics Association: JAMIA, 19(3), 328333. https://doi.org/10.1136/amiajnl-2011-000394.

Furukawa, M. F., Patel, V., Charles, D., Swain, M., \& Mostashari, F. (2013). Hospital electronic health information exchange grew substantially in 2008-12. Health Aff (Millwood), 32(8), 1346-1354. https://doi.org/10.1377/hlthaff.2013.0010.

Gadd, C. S., Ho, Y.-X., Cala, C. M., Blakemore, D., Chen, Q., Frisse, M. E., et al. (2011). User perspectives on the usability of a regional health information exchange. JAMIA, 18(5), 711-716.

Genes, N., Shapiro, J., Vaidya, S., \& Kuperman, G. (2011). Adoption of health information exchange by emergency physicians at three urban academic medical centers. Applied Clinical Informatics, 2(03), 263-269.

Haidar, Y. M., Moshtaghi, O., Mahboubi, H., Ghavami, Y., Ziai, K., Hojjat, H., et al. (2017). Association between electronic medical record implementation and otolaryngologist productivity in the ambulatory setting. JAMA Otolaryngology-Head \& Neck Surgery 143(1), 20-24.

Hennington, A., \& Janz, B. D. (2007). Information systems and healthcare XVI: Physician adoption of electronic medical records: Applying the UTAUT model in a healthcare context. Communications of the Association for Information Systems, 19(1), 5.

Hessler, B. J., Soper, P., Bondy, J., Hanes, P., \& Davidson, A. (2009). Assessing the relationship between health information exchanges and public health agencies. Journal Of Public Health Management And Practice: JPHMP, 15(5), 416-424. https://doi.org/10.1097/01. PHH.0000359636.63529.74
Hincapie, A., \& Warholak, T. (2011). The impact of health information exchange on health outcomes. Applied Clinical Informatics, 2(4), 499-507. https://doi.org/10.4338/ACI-2011-05-R-0027.

Hu, P. J., Chau, P. Y., Sheng, O. R. L., \& Tam, K. Y. (1999). Examining the technology acceptance model using physician acceptance of telemedicine technology. Journal of Management Information Systems, 16(2), 91-112.

Jackson, C. M., Chow, S., \& Leitch, R. A. (1997). Toward an understanding of the behavioral intention to use an information system. Decision Sciences, 28(2), 357-389.

Jasperson, J. S., Carter, P. E., \& Zmud, R. W. (2005). A comprehensive conceptualization of post-adoptive behaviors associated with information technology enabled work systems. MIS Quarterly, 29(3), $525-557$.

Kaelber, D. C., \& Bates, D. W. (2007). Health information exchange and patient safety. Journal of Biomedical Informatics, $40(6$ Suppl), S40-S45.

Kaminski, J. (2011). Diffusion of innovation theory. Canadian Journal of Nursing Informatics, 6(2), 1-6.

Lee, S.-i., Park, H., Kim, J.-W., Hwang, H., Cho, E.-Y., Kim, Y., et al. (2012). Physicians' perceptions and use of a health information exchange: A pilot program in South Korea. Telemedicine and eHealth, 18(8), 604-612.

Melvin, C. L., Saef, S. H., Pierce, H. O., Obeid, J. S., \& Carr, C. M. (2016). Health information exchange in the ED: What do ED clinicians think? Southern Medical Journal, 109(7), 419.

Paré, G., Sicotte, C., \& Jacques, H. (2006). The effects of creating psychological ownership on physicians' acceptance of clinical information systems. Journal of the American Medical Informatics Association, 13(2), 197-205.

Patel, V., Abramson, E. L., Edwards, A., Malhotra, S., \& Kaushal, R. (2011). Physicians' potential use and preferences related to health information exchange. International Journal of Medical Informatics, 80(3), 171-180. https://doi.org/10.1016/j.ijmedinf. 2010.11.008

Rahurkar, S., Vest, J. R., \& Menachemi, N. (2015). Despite the spread of health information exchange, there is little evidence of its impact on cost, use, and quality of care. Health Affairs, 34(3), 477-483. https:// doi.org/10.1377/hlthaff.2014.0729.

Richardson, W. C. (2001). Crossing the quality chasm: A new health system for the 21st century. Washington, DC: Institute of Medicine.

Ross, S. E., Schilling, L. M., Fernald, D. H., Davidson, A. J., \& West, D. R. (2010). Health information exchange in small-to-medium sized family medicine practices: Motivators, barriers, and potential facilitators of adoption. International Journal of Medical Informatics, 79(2), 123-129. https://doi.org/10.1016/j.ijmedinf.2009.12.001.

Ross, S. E., Radcliff, T. A., LeBlanc, W. G., Dickinson, L. M., Libby, A. M., \& Nease Jr., D. E. (2013). Effects of health information exchange adoption on ambulatory testing rates. Journal of the American Medical Informatics Association, 20(6), 1137-1142.

Rudin, R., Volk, L., Simon, S., \& Bates, D. (2011). What affects clinicians' usage of health information exchange? [article]. Applied Clinical Informatics, 2(3), 250-262. https://doi.org/10.4338/ACI2011-03-RA-0021.

Rudin, R. S., Motala, A., Goldzweig, C. L., \& Shekelle, P. G. (2014). Usage and effect of health information exchange: A systematic review. Annals of Internal Medicine, 161(11), 803-811. https://doi. org/10.7326/M14-0877.

Shade, S. B., Chakravarty, D., Koester, K. A., Steward, W. T., \& Myers, J. J. (2012). Health information exchange interventions can enhance quality and continuity of HIV care. International Journal of Medical Informatics, 81(10), e1-e9. https://doi.org/ 10.1016/j.ijmedinf.2012.07.003.

Shapiro, J. S., Mostashari, F., Hripcsak, G., Soulakis, N., \& Kuperman, G. (2011). Using health information exchange to improve public 
health. American Journal of Public Health, 101(4), 616-623. https://doi.org/10.2105/ajph.2008.158980.

Taylor, S., \& Todd, P. (1995a). Assessing IT usage: The role of prior experience. MIS Quarterly, 561-570.

Taylor, S., \& Todd, P. A. (1995b). Understanding information technology usage: A test of competing models. Information Systems Research, 6(2), 144-176.

Thompson, R. L., Higgins, C. A., \& Howell, J. M. (1994). Influence of experience on personal computer utilization: Testing a conceptual model. Journal of Management Information Systems, 11(1), 167187.

Triandis, H. C. (1977). Interpersonal behavior. Monterey: Brooks/Cole Publishing Company.

Tukey, J. W. (1949). Comparing individual means in the analysis of variance. Biometrics, 5(2), 99-114.

Unertl, K., Johnson, K., Gadd, C., \& Lrenzi, N. (2013). Bridging organizational divides in healthcare: An ecological view of health information exchange. JMIR Medical Informatics, 1(1), e3. https://doi. org/10.2196/medinform.2510.

Vaidya, S. R., Shapiro, J. S., Papa, A. V., Kuperman, G., Ali, N., Check, T., et al. (2012). Perceptions of health information exchange in home healthcare. CIN: Computers, Informatics, Nursing, 30(9), 503-509.

Vest, J. R., \& Miller, T. R. (2011). The association between health information exchange and measures of patient satisfaction. Applied Clinical Informatics, 2(4), 447-459. https://doi.org/10.4338/ACI2011-06-RA-0040.

Vest, J. R., Zhao, H., Jaspserson, J., Gamm, L. D., \& Ohsfeldt, R. L. (2011). Factors motivating and affecting health information exchange usage. Journal of the American Medical Informatics Association, 18(2), 143-149. https://doi.org/10.1136/jamia.2010. 004812 .

Yaraghi, N. (2015). An empirical analysis of the financial benefits of health information exchange in emergency departments. Journal of the American Medical Informatics Association, 22(6), 11691172. https://doi.org/10.1093/jamia/ocv068.

Zech, J., Husk, G., Moore, T., Kuperman, G. J., \& Shapiro, J. S. (2015). Identifying homelessness using health information exchange data. Journal of the American Medical Informatics Association, 22(3), 682-687.

Publisher's Note Springer Nature remains neutral with regard to jurisdictional claims in published maps and institutional affiliations.

Sue Feldman, RN, MEd, PhD is Professor and Director of Graduate Programs in Health Informatics in the School of Health Professions at the University of Alabama at Birmingham. Dr. Feldman is also a Senior Scientist in the Informatics Institute and Senior Fellow in the Center for the Study of Community Health. Her research focuses on health information systems - from development to evaluation. Dr. Feldman also serves on the Health Informatics Accreditation Council for the Commission on
Accreditation for Health Informatics and Information Management (CAHIIM), chairs Graduate Health Informatics accreditation site visit teams, and has studied and developed graduate level health informatics curriculum. As a Registered Nurse (RN) for over 30 years, she brings a unique clinical and informatics blend to everything she does, grounding policy and theory with practice. Dr. Feldman has published in a variety of top-tier peer-reviewed journals and conference proceedings, led or co-led the development of several information systems that are grounded in research, and has served as program chair for several national forums. Her current work involves leading the development of a substance use, abuse, and recovery data collection system for the state of Alabama as well as a statewide COVID-19 symptom and exposure notification system. Dr. Feldman has a Masters degree in Education and a $\mathrm{PhD}$ in Education and also in Information Systems and Technology from Claremont Graduate University.

Neşet Hikmet is Professor of Health Information Technology in the College of Engineering and Computing, University of South Carolina where he also serves as the Director of Applied Sciences at Center for Applied Innovation and Advanced Analytics. His research expertise includes cloud computing, augmented analytics, health informatics, and healthcare internet of things. As an applied scientist he is heavily involved in design, development, deployment, and maintenance of large-scale computing systems. Dr. Hikmet has significant experience in leading health data analytics projects within academia, including utilization of a wide range of analytics methods and approaches. His research and projects have been funded by the National Science Foundation, National Institutes of Health, and other Federal and State agencies and Private Foundations

Shikha Modi, MBA is a graduate of UAB's MBA program with an emphasis on healthcare services. Ms. Modi has a Bachelor's degree in Biology from University of North Alabama. Ms. Modi's current research projects include Health information technology and its impact on outcomes, health information exchange and provider performance, health information exchange and patient and population health, the intersection of health informatics, healthcare quality and safety, and healthcare simulation, patient experience evaluation at dermatology clinics, and identifying and mitigating bias in artificial intelligence systems.

Benjamin Schooley, MBA, PhD is Associate Professor of Health IT in the College of Engineering and Computing, University of South Carolina where he also serves as Research Director at the Health Information Technology Consortium. His research expertise includes human-computer interaction, health informatics, and human factors in the design and application of software systems. As a design scientist, his applied and field research in health, wellness and social-benefit initiatives have been funded by the National Science Foundation, National Institutes of Health, The Centers for Medicaid and Medicare Services, the U.S. Department of Labor, Social Security Administration, and other Federal and State agencies and Private Foundations. 